\title{
COVID-19 Stay-at-Home Orders Result in a Decrease in the Number of Missing Daily Precipitation Observations
}

\author{
Jessica Spaccio, Arthur DeGaetano, and Nolan Doesken
}

\begin{abstract}
$\mathrm{n}$ the early spring of 2020, the COVID-19 pandemic began to impact the United States. As cases, hospitalizations, and deaths increased, states began issuing stay-at-home recommendations or orders. According to New York Times reports, by 23 March, 20 states had issued orders; by 26 March, 35 states had issued orders; and by 30 March, 43 states had issued such orders. The Center for Disease Control (CDC) issued a domestic travel advisory for New York,
\end{abstract}

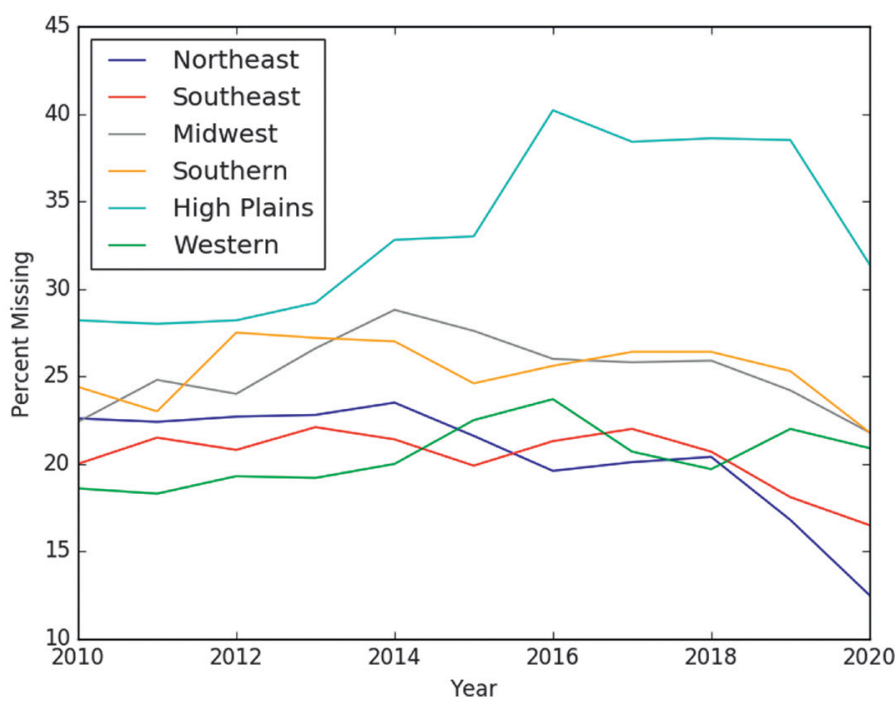

Fig. 1. Time series of the percent of missing daily CoCoRaHS observations for the period 15 Mar-30 Apr 2020, by Regional Climate Center region.

New Jersey, and Connecticut. Under these recommendations, many Americans were not leaving their homes.

Such changes in daily routines have the potential to affect the reporting of daily weather observations at U.S. stations that are a part of the Global Historical Climate Network (GHCN). GHCN observations are primarily taken by volunteer observers either at their residences or workplaces. Nearly 23,000 GCHN observations are recorded across the United States each day. Given the volunteer nature of the network, missing observations are an inevitable part of the data record as observers are often unable to take daily measurements given travel, sickness, or other competing responsibilities. In other cases, when the observing site is at a place of employment, such as a university research farm or water utility, weekend observations are often missing given the typical work week schedule of the employee(s) tasked with making the observation. At such sites, work-from-home requirements may have increased the number of missing data reports. Alternatively, it is possible that data reports for stations located at observers' residences experienced a decrease in missing entries.

The GHCN merges data from a number of independent data networks. Two networks account for the majority of U.S. observations. Approximately a third of daily U.S. GHCN observations are from the NOAA National Weather Service (NWS) Cooperative Observer Program (COOP) network. COOP, which was formally created in 1890 , is overseen by the NWS. Observers are both individuals and organizations. Daily observations of maximum and minimum temperatures, snowfall, snow depth, and precipitation are taken using NWS-approved equipment. While different gauges are used to measure precipitation, an 8-in. standard rain gauge is preferred. The gauge consists of an openmouth can with straight sides, collector funnel, and an inner measuring tube. When frozen or freezing precipitation

AfFiliations: Spaccio And Degaetano-Northeast Regional Climate Center, Cornell University, Ithaca, New York; DoESKENCoCoRaHS, Colorado State University, Fort Collins, Colorado

CORRESPONDING AUTHOR: Dr. Art DeGaetano, atd2@cornell.edu DOI:10.1175/BAMS-D-20-0170.1

In final form 25 August 2020

C2021 American Meteorological Society

For information regarding reuse of this content and general copyright information, consult the AMS Copyright Policy. 

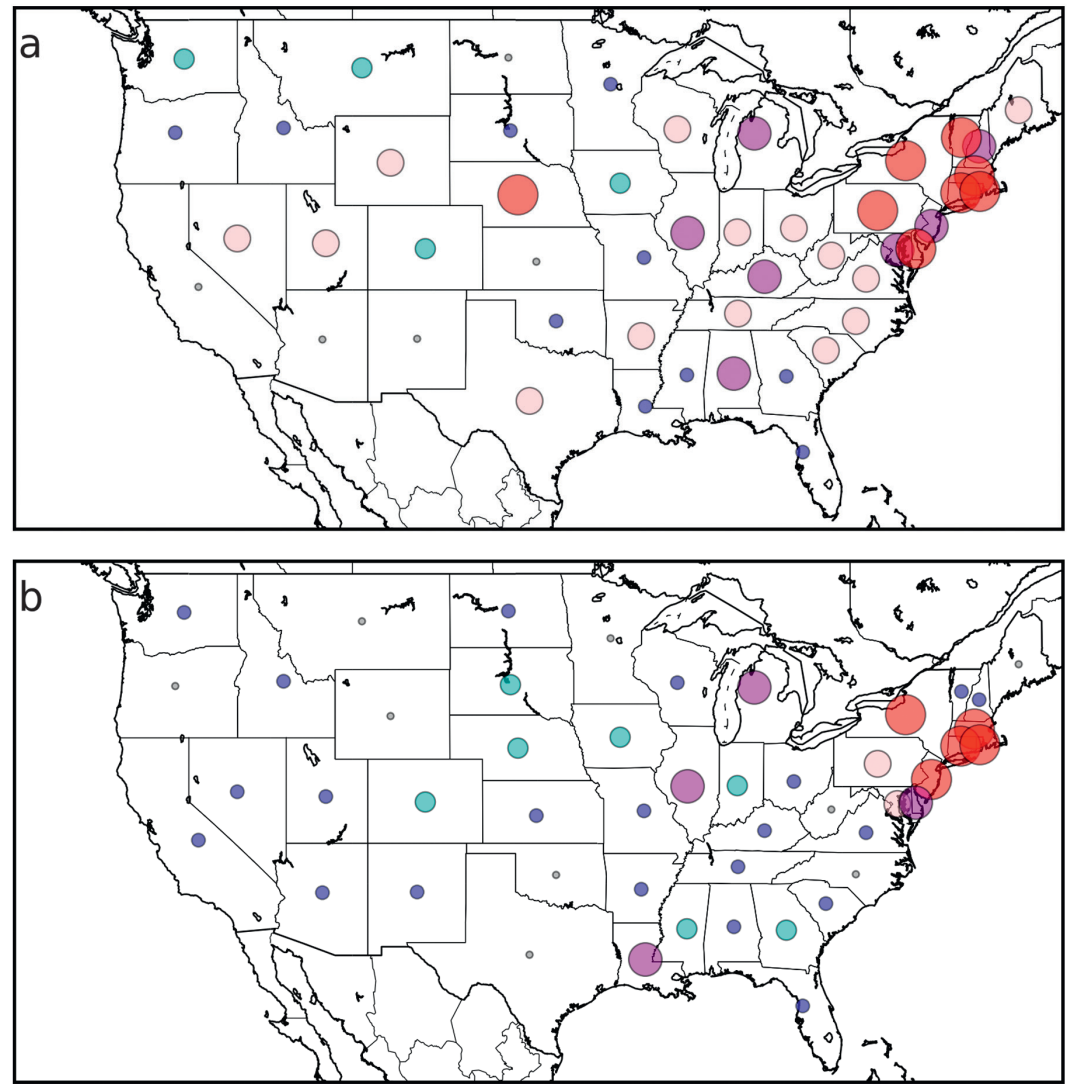

Fig. 2. Maps of (a) percentage of missing CoCoRaHS observations relative to the 2010-19 average and (b) active COVID-19 cases by state. In (a), a larger circle radius indicates fewer missing observations, with the largest red circles indicating a $>8 \%$ decrease in missing observations, while the smallest circles indicate states with an increase in missing observations. In (b), larger circles indicate a higher number of COVID-19 cases, with more than 750 cases per $10^{5}$ residents denoted by the largest red circles and fewer than 100 cases per $10^{5}$ residents denoted by the smallest circles.

is expected, the funnel and inner tube are removed. The frozen contents are melted and poured into the measuring tube. Instrument siting and performance must meet NWS standards, and observers are required to complete training.

More recently, the GHCN has included rainfall observations from the Community Collaborative Rain, Hail and Snow (CoCoRaHS) network. In 2019, approximately 66\% of U.S. daily GHCN observations came from CoCoRaHS. The CoCoRaHS network is composed entirely of individual volunteers who take observations primarily at their homes; however, school and business locations are also a part of the network. CoCoRaHS has been in operation since 1998 As the CoCoRaHS network observes rain, hail, and snow, the parameters of this analysis were limited to precipita- tion. Precipitation, rainfall, and/or the liquid equivalent of snowfall, is measured using a 4-in. plastic rain gauge.

Observations from both networks are submitted primarily online. COOP observers submit their data through the WxCoder system, the official webbased entry system for the program that allows users to submit their daily observations online as well as view and edit previous observations. CoCoRaHS observers submit their data through the CoCoRaHS network website or via smartphone apps. Observations from both networks are taken once per day at a specified time.

To assess how the COVID-19 stayat-home orders affected the number of missing GHCN observations, the Applied Climate Information System (ACIS) was used to access GHCN observations from all stations within each state during the period from 15 March through 30 April 2020. ACIS metadata record the base network associated with each station, allowing CoCoRaHS observations to be counted separately. Non-CoCoRaHS observations were analyzed separately and are referred to here as COOP observations, given these values mainly came from that network.

Based on the counts of missing data at each station during the 47-day quarantine period, a percentage of missing observations values, $P_{\text {miss }}$, was computed for each state based on the equation

$P_{\text {miss }}=\frac{N_{\text {miss }}}{47\left(N_{\text {station }}\right)}$.

In the equation, $N_{\text {miss }}$ is the count of missing data values at all active stations within a state $\left(N_{\text {station }}\right)$, and 47 represents the number of days from 15 March to 30 April. Active stations were required to have reported at least one observation from 15 March to 30 April.

To place each state's $2020 P_{\text {miss }}$ value into historical context, $P_{\text {miss }}$ was also computed for each year from 2010 to 2019. While observations from both networks exist prior to 2010, some states did not have active CoCoRaHS observers 
before 2010. The state data were also aggregated into the six Regional Climate Center regions. The missing counts and total possible observations were summed for all the states within a region, and the percent missing observations were calculated from those values.

Figure 1 shows time series of $P_{\text {miss }}$ for each region. The Northeast and Southeast are notable In that the percentage of missing CoCoRaHS observations attain their lowest levels of the decade in 2020. In the Northeast, only $12 \%$ of the possible daily precipitation observations were missing during 15 March-30 April 2020. Typically, more than 20\% of the possible observations are missing at Northeast and Southeast stations during this time period. In 2020, 16\% of the possible Southeast CoCoRaHS precipitation observations are missing.

To a lesser degree, the $2020 P_{\text {miss }}$ is also among the lowest of the values recorded during the last decade in the Midwest and Southern regions. These regions typically report more missing values than the Southeast and Northeast, with about a quarter of the possible values missing (Fig. 1). From 15 March to 30 April 2020, only 22\% of the possible observations were missing. In the Western and Great Plains regions, $P_{\text {miss }}$ in 2020 did not vary greatly from the historical average.

In terms of individual states, $P_{\text {miss }}$ from the CoCoRaHS network was lower for many states during 15 March30 April 2020 than the same time period in the previous years. Arkansas, Colorado, Connecticut, Hawaii, Illinois,

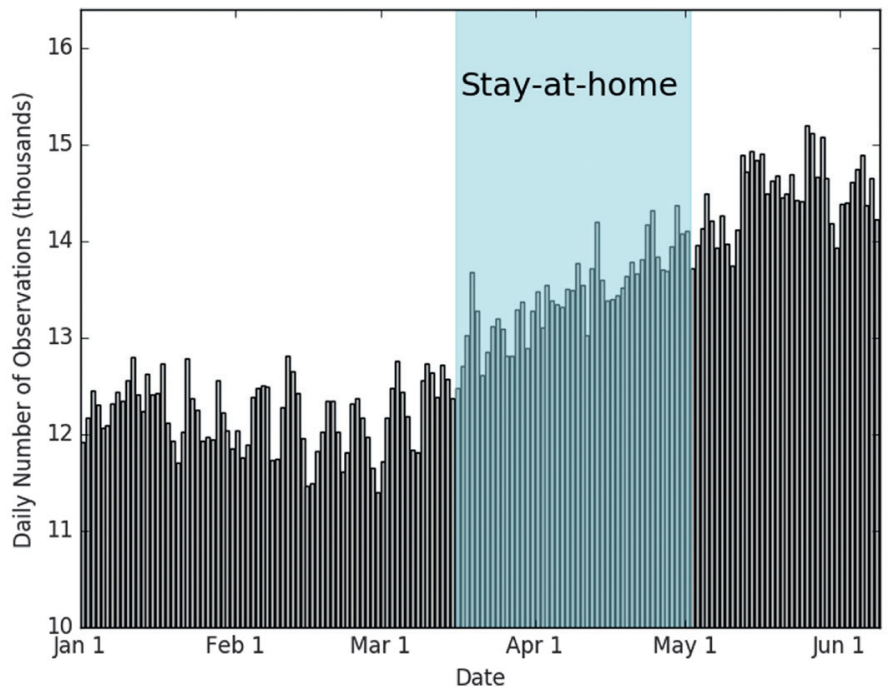

Fig. 3. Number of daily CoCoRaHS precipitation reports for the period 1 Jan-10 Jun 2020.
Indiana, Kentucky, Maryland, Massachusetts, Michigan, New Hampshire, New Jersey, New York, North Carolina, Ohio, Pennsylvania, Rhode Island, South Carolina, Tennessee, Texas, Vermont, Virginia, and Wyoming all saw the least $P_{\text {miss }}$ in 2020. This was most evident in eastern states that were greatly affected by COVID-19 during this period. Figure 2a depicts the difference between the 2010-19 $P_{\text {miss }}$ average and the 2020 value by state for CoCoRaHS stations. The larger circles identify states that had fewer missing observations in 2020. Cumulative incidence rates of COVID-19 cases per 100,000 residents as of 30 April 2020 based on data reported by the Centers for Disease Control's COVID Data Tracker are shown in Fig. 2b. There is strong correspondence between the states with the highest number of COVID-19 cases and the fewest missing CoCoRaHS observations. This suggests that as more observers stayed home, they were more apt to routinely take and report their precipitation observations.

Figure 3 shows a time series of the total number of daily CoCoRaHS reports during the first 162 days of 2020. During the stay-at-home period, the number of CoCoRaHS reports increased by $25 \%$, from approximately 12,000 daily reports during January through early March to nearly 15,000 reports at the end of May. CoCoRaHS daily reports usually increase each spring, but the 2020 increase was particularly strong. Another feature that stands out during the spring of 2020 is the pronounced change in the weekly reporting cycle. Throughout the history of CoCoRaHS, there has been a clear weekly cycle in reporting, with the number of reports increasing on weekdays and decreasing on weekends. As widespread stay-at-home orders commenced in mid-March, the amplitude of this weekly cycle decreased, with fewer missing weekend observations (Fig. 3). Thus, the decline in missing observations seen in Fig. 1 reflects this decrease in missing weekend observations. Starting in late May, the number of total CoCoRaHS observations began to decline, presumably in response to the relaxation of stay-at-home restrictions across the country. Likewise at the same time, a more pronounced weekly cycle in the number of observations began to reappear.

Similar analyses based on the COOP network showed more varied results. Some states had a slight increase of percent of missing observations while others a slight decrease. In no cases was the $2020 P_{\text {miss }}$ value for COOP stations notably different from the 2010-19 average. 